\title{
Presence of Coronary Artery Disease in Patients on Methadone in the South Bronx: A Retrospective Analysis and Literature Review
}

\author{
Rodríguez-Guerra, Miguel MD ${ }^{\text {*1}}$; Urlapu, Kinnera Sahithi MD ${ }^{1}$; Hewapathirana, Udanie ${ }^{3}$; Vikram, \\ Itare MD ${ }^{1}$; Fortuzi, Ked MD ${ }^{1}$; Mendoza, Christian MD ${ }^{1}$; Shah, Niel MD ${ }^{1}$; Vittorio, Timothy MD ${ }^{2}$ \\ ${ }^{1}$ BronxCare Hospital Center, Department of Internal Medicine, Bronx, NY, USA \\ ${ }^{2}$ BronxCare Hospital Center, Division of Cardiology, Bronx, NY, USA \\ ${ }^{3}$ American University of the Caribbean School of Medicine, Rotating at BronxCare Hospital Center \\ *Corresponding author: Rodríguez-Guerra, Miguel MD; m.rodriguezg.md@gmail.com
}

Received 21 February 2021; $\quad$ Accepted 26 March 2021; $\quad$ Published 01 April 2021

\begin{abstract}
Introduction: The South Bronx community has faced a challenge with the addiction to opioid, long-acting medication like Methadone have been proved to be helpful in the setting of withdrawal, rehab, detox or chronic pain management, but also represent risk of dependence. Besides most of the literature expose the relation between opioid could be related to cardiac disease, there are also reports relating methadone with a possible positive impact in coronary artery disease. Method: Retrospective and descriptive review of records and literature review. $\underline{\text { Result: }}$ Total of 207 of patients on methadone had coronary artery disease (CAD), of which 128 pts were known to have CAD from before, of this group the most common group was Hispanic between 45-64 years old. Regarding the cardiovascular (CV) risks, the 89.06\% (114 pts) were hypertensive, $61.7 \%$ (79 pts) had dyslipidemia, $60.2 \%$ (77pts) were diabetic, $24.21 \%$ (31pts) had Kidney disease with CKD III or higher. $7 \%$ (9 pts) were known to have prolonged QTc, while $4.68 \%$ (6 pts) were newly diagnosed with it (Figure 1). When we studied ejection fraction (EF), in the known CAD patients, we observed that the $20 \%$ (26 pts) had EF < 40\%, while the $10.16 \%$ (13 pts) had intermediate EF (40-55\%). Over the study period 97 pts had follow up echocardiography of which $17.52 \%$ (17 pts) had improved EF, 64.94\% (63pts) had no change in EF, while $17.52 \%$ (17 pts) had decreased EF. Regarding the cardiac events in this group, 13 patients had myocardial infarctions, of which six patients had ejection fraction below $40 \%$ and one patient had prolonged QTc (Figure 2). Only $9.38 \%$ of the patients (12) had a follow up cardiac catheterization, of which $83 \%$ (10 pts) showed a progression of CAD. In the other hand, a total 38.16\% (79 pts) had newly diagnosed with CAD, of which $62 \%$ (49 pts) were hypertensive, $37.97 \%$ (30 pts) had diabetes and $117.72 \%$ (14 pts) had CKD III or more. A total of $12.66 \%$ (10 pts) were known to have prolonged QTc while 6.33\% (5 pts) were newly diagnosed with it. The $13.9 \%$ (11 pts) were found to have an improvement in ejection fraction while the $11.4 \%$ (9 pts) got worse. Two of these patients had myocardial infarction. (Figure 3). Follow up echocardiograms showed that the ejection fraction did not worsen in 90 out of 116 patients. Overall, only $1.93 \%$ (4 pts) had cardiac arrest of which 3 pts had prior history of CAD and none of them had prolonged QTc. Conclusion: This study has exposed the possibility of the positive effect of methadone in the cardiac pump function. There appears to be a progression of CAD in our patients who underwent to cardiac catheterization as a follow up, but due to the size of the sample of patient we cannot establish this relation as the definitive risk for the progression of the disease. Based on the literature review and our results, there is no doubt in the possible potential positive effect that long term use of opioids could have or maybe the negative cardiovascular effect, from the cardiac point of view. The incidence of fatal events did not represent a higher risk (Arrhythmias, myocardial infarction, or cardiac arrest) than the potential benefit (Improvement of heart function or mitigation of CAD).
\end{abstract}

Keywords: Methadone, Coronary Artery Disease, Opioid

\section{Introduction}

Addiction to opioid medications has been a challenge to our community ${ }^{[1]}$, multiple elements ranging from chronic pain to substance dependency seems to be contributing to this ${ }^{[2]}$. To combat the unsupervised use of opioids, long-acting medication like Methadone has been the drug of choice to manage patients suffering from substance dependence by trying to taper the doses ${ }^{[3]}$.

In our community, the South Bronx, the incidence of recreational drugs, including opioids is significantly high, as per Department of Health (DOH) in the last decade, the number of deaths due to opioid overdose increased by $200 \%$, in spite of the 
investment of 7 million dollars in prevention of opioid overdosing [4]. In addition to the high number of patients in supervised Methadone programs in the south Bronx, there is also a huge group of patients who use unregulated or unsupervised methadone obtained from other non-controlled sources.

Methadone is a powerful medication that could be helpful in the setting of withdrawal ${ }^{[5]}$, rehab ${ }^{[6]}$, detox or chronic pain management ${ }^{[7]}$, but at the same time, the risk of dependence is high ${ }^{[8]}$. So supervised methadone programs which closely follow the patients. On the other hand, those patients who obtain it from external sources are at high risk of dependence and sometimes have fatal side effects.

Review of medical literature shows that this medication has been associated with multiple cardiovascular abnormalities including but not limited to arrhythmias, pre-mature vascular disease and heart dysfunction ${ }^{[9]}$. Most of the studies reports the negative impact of Methadone in the cardiovascular system, however there are a few studies that brought out the possibility of a positive impact such as cardio protective effect against CAD, in terms of size of infarction, ischemic events related arrhythmias; this article looks towards understanding the incidence of CAD (coronary Artery Disease) among methadone users in the South Bronx ${ }^{[10,11]}$, here we discuss a cautious retrospective analysis and literature review about the use of methadone in patients with coronary artery disease, their disease severity and outcome in terms of mortality.

\section{Objectives}

- Establish the correlation between methadone and coronary artery disease in our patient population from the South Bronx.

- Define the mortality risk in CAD patients associated with methadone use

\section{Method}

This is a retrospective and descriptive study comparing patients on methadone with history of coronary artery disease. The study population is patients(pts) at the BronxCare Health System from 2010 - 2019; socio demographic and clinical data was collected and reviewed. Process of study was: MD Questionnaire was applied to our patients who met our inclusion criteria. Tabulation was done using Microsoft Access 2007, Microsoft Excel and later was exported to SPSS program for calculations and statistical tables. ANOVA was used with a $\mathrm{p}=0.000$. Literature review of 30 original articles found in multiple medical journals using the keyword methadone, opioid, coronary artery disease, and their combinations.

\section{Inclusion criteria}

Adult patients (older than 18 years of age), who use methadone and history of coronary artery disease in the BronxCare Health System in the last 10 years.

\section{Exclusion criteria}

Non-adult patients or patients without cardiac ischemia work up.

\section{Results}

Total of 207 of patients on methadone had coronary artery disease (CAD), of which 128 pts were known to have CAD from before, of this group the most common group was Hispanic between 45-64 years old. Regarding the cardiovascular (CV) risks, the $89.06 \%$ (114 pts) were hypertensive, 61.7\% (79 pts) had dyslipidemia, $60.2 \%$ (77pts) were diabetic, $24.21 \%$ (31pts) had Kidney disease with CKD III or higher. $7 \%$ (9 pts) were known to have prolonged QTc, while 4.68\% (6 pts) were newly diagnosed with it (Figure 1).

When we studied ejection fraction (EF), in the known CAD patients, we observed that the $20 \%$ (26 pts) had EF < 40\%, while the $10.16 \%$ (13 pts) had intermediate EF (40-55\%). Over the study period 97 pts had follow up echocardiography of which $17.52 \%$ (17 pts) had improved EF, 64.94\% (63pts) had no change in EF, while $17.52 \%$ (17 pts) had decreased EF. Regarding the cardiac events in this group, 13 patients had myocardial infarctions, of which six patients had ejection fraction below $40 \%$ and one patient had prolonged QTc (Figure 2).

Only $9.38 \%$ of the patients (12) had a follow up cardiac catheterization, of which $83 \%$ (10 pts) showed a progression of CAD.

In the other hand, a total $38.16 \%$ (79 pts) had newly diagnosed with $\mathrm{CAD}$, of which $62 \%$ (49 pts) were hypertensive, $37.97 \%$ (30 pts) had diabetes and $117.72 \%$ (14 pts) had CKD III or more. A total of $12.66 \%$ (10 pts) were known to have prolonged QTc while $6.33 \%$ (5 pts) were newly diagnosed with it. The $13.9 \%$ (11 pts) were found to have an improvement in ejection fraction while the $11.4 \%$ (9 pts) got worse. Two of these patients had myocardial infarction. (Figure 3).

Follow up echocardiograms showed that the ejection fraction did not worsen in 90 out of 116 patients.

Overall, only $1.93 \%$ (4 pts) had cardiac arrest of which 3 pts had prior history of CAD and none of them had prolonged QTc.

\section{History of CAD}

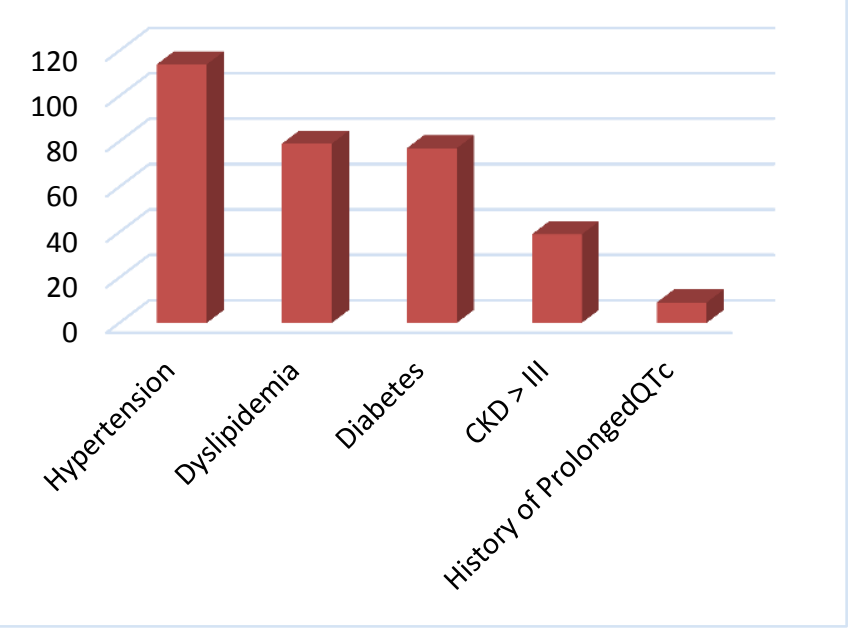

Figure 1 


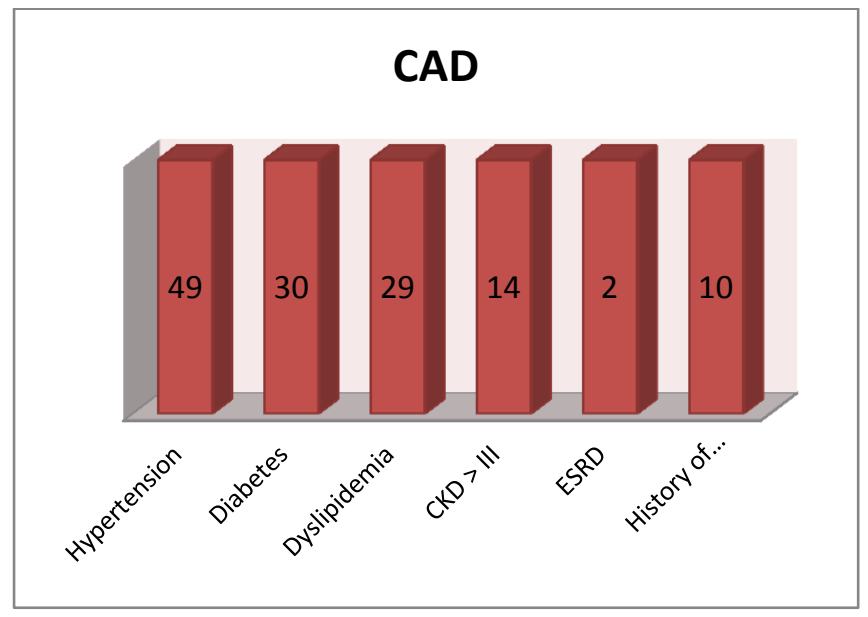

Figure 2

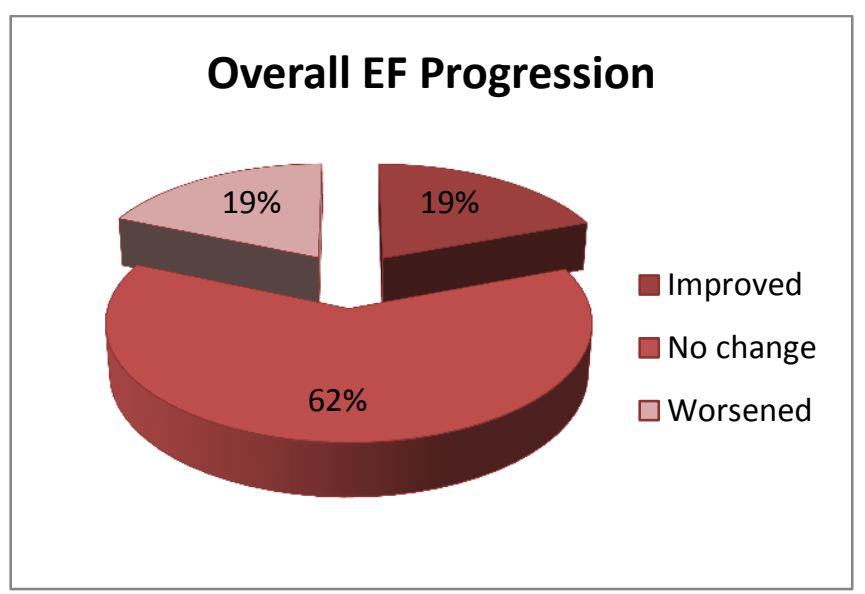

Figure 3

\section{Discussion}

Opioid addiction was one of the most arduous challenges the medical community has been facing, due to the high impact in the cost and complications that its consequences could represent ${ }^{[12,13]}$. As part of the solution, multiple medications with a longer half-life (like methadone or naltrexone) were used to try to wean the patients of their addiction, but these alternatives are not free of potential side effects neither complication of comorbidities in this population $^{[14,15]}$.

The use of methadone has been related to multiple cardiovascular affections like electrocardiography abnormalities (Especially prolongation of the QTc) ${ }^{[16,17]}$, predisposition to cardiac arrhythmias, atherosclerosis including coronary artery disease, and sudden cardiac death ${ }^{[18,19,20]}$.

The activation of opioid, adenosine, bradykinin, adrenergic, and other G-protein coupled receptors are cardioprotective ${ }^{[21]}$. It is known that the $\kappa$ - and/or $\delta$-opioid receptor activation is involved in direct myocardial protection, while the role of $\mu$-opioid receptors seems less clear ${ }^{[22]}$; multiple theories about the advantages or disadvantages of the methadone were proposed in the literature $[23,24,25]$.

For example, a study from Iran, suggested the positive effect of opiates in cardiovascular diseases including CAD but most of them mentioned that the results could be due to a confusion bias ${ }^{[26]}$. In Greece, multiple studies have shown the positive effect of orally administered opiates (including methadone) in ischemic cerebrovascular events but no major effect in ischemic heart events.
While many animal studies were done to identify the opioid related complications from the cardiac point of view; most of the studies and reviews showed a potential positive effect in terms of improvement of the reperfusion ischemia and also decreasing the size of the MI when the medication is given during the event ${ }^{[27]}$.

In the retrospective analysis of 5,227 autopsies, Michael Marmor et al. in coordination with the Office of the Chief Medical Examiner of New York City reported that long-term opiate exposure may mitigate $\mathrm{CAD}$ severity and its often-fatal consequences ${ }^{[28]}$.

Multiple cases reported the relation between opioid withdrawal and takotsubo cardiomyopathy ${ }^{[29,30]}$, but in our retrospective review of medical record, we did not find evidence for this correlation.

\section{Conclusion}

In conclusion, coronary artery disease in patients on methadone therapy was more common in Hispanics between 45-64 years old and the most common cardiovascular risk factors were hypertension followed by diabetes.

Out of the study population, only twelve patients had cardiac catheterization follow-up, most of the patients presented a progression of the disease; the population of patients studied showed a significant improvement of the ejection fraction. The use of methadone was not related to a significant prolongation of the QTc or cardiac arrest.

This study has exposed the possibility of the positive effect of methadone in the cardiac pump function. There appears to be a progression of CAD in our patients who underwent to cardiac catheterization as a follow up, but due to the size of the sample of patient we cannot establish this relation as the definitive risk for the progression of the disease.

Based on the literature review and our results, there is no doubt in the possible potential positive effect that long term use of opioids could have from the cardiac point of view. The incidence of fatal events did not represent a higher risk (Arrhythmias, myocardial infarction, or cardiac arrest) than the potential benefit (Improvement of heart function or mitigation of CAD).

Randomized, prospective studies with established followup would be needed to confirm these results and to define the impact of methadone in the progression of coronary artery disease.

\section{Ethics approval and consent to participate}

This is a retrospective review of records, approved by our Institutional Review Board.

\section{Data Availability}

The actual information is available in MR system of our hospital

\section{Conflicts of Interest}

The authors declare that there is no conflict of interest regarding the publication of this paper.

\section{Funding Statement}

Authors did not receive financial support for this study.

\section{Authors' contributions}


All authors contributed equally to this study and also read and approved the final manuscript.

\section{References}

[1] Katz, N. P., Birnbaum, H., Brennan, M. J., Freedman, J. D., Gilmore, G. P., Jay, D., Kenna, G. A., Madras, B. K., McElhaney, L., Weiss, R. D., \& White, A. G. (2013). Prescription opioid abuse: challenges and opportunities for payers. The American journal of managed care, 19(4), 295-302.

[2] Premila Devi, J., Azriani, A. R., Zahiruddin, W. M., Mohd Ariff, M. N., \& Noor Hashimah, A. (2012). The Effectiveness of Methadone Maintenance Therapy Among Opiate - Dependants Registered with Hospital Raja Perempuan Zainab II Kota Bharu, Kelantan. The Malaysian journal of medical sciences: MJMS, 19(4), 17-22.

[3] Breslin, K. T., \& Malone, S. (2006). Maintaining the viability and safety of the methadone maintenance treatment program. Journal of psychoactive drugs, 38(2), $157-160$.

https://doi.org/10.1080/02791072.2006.10399840

[4] Rose A. Rudd et al. Increases in Drug and Opioid Overdose Deaths - United States, 2000-2014. January 1, 2016 / 64(50);1378-82. On December 18, this report was posted as an MMWR Early Release on the MMWR website (http://www.cdc.gov/mmwr).

[5] Amato, L., Davoli, M., Ferri, M., \& Ali, R. (2003). Methadone at tapered doses for the management of opioid withdrawal. The Cochrane database of systematic reviews,

CD003409. https://doi.org/10.1002/14651858.CD003409

[6] Wakeman SE, Larochelle MR, Ameli O, et al. Comparative Effectiveness of Different Treatment Pathways for Opioid Use Disorder. JAMA Netw Open. 2020;3(2):e1920622.

doi:10.1001/jamanetworkopen.2019.20622

[7] Hsu DJ, McCarthy EP, Stevens JP, Mukamal KJ. Hospitalizations, costs and outcomes associated with heroin and prescription opioid overdoses in the United States 2001-12. Addiction. 2017;112(9):1558-1564. doi:10.1111/add.13795

[8] Grella, C. E., Anglin, M. D., Wugalter, S. E., Rawson, R., \& Hasson, A. (1994). Reasons for discharge from methadone maintenance for addicts at high risk of HIV infection or transmission. Journal of psychoactive drugs, 26(2), 223-232. https://doi.org/10.1080/02791072.1994.10472270

[9] Lea C. Price et al. Methadone for Pain and the Risk of Adverse Cardiac Outcomes. Journal of Pain and Symptoms Management. DOI: https://doi.org/10.1016/j.jpainsymman.2013.09.021

[10] Fox, A. D., Shah, P. A., Sohler, N. L., Lopez, C. M., Starrels, J. L., \& Cunningham, C. O. (2014). I heard about it from a friend: assessing interest in buprenorphine treatment. Substance abuse, 35(1), 74-79. https://doi.org/10.1080/08897077.2013.804484

[11] Brown, L. S., \& Kritz, S. (2017). Methadone Misinformation and Misconceptions. American journal of public health, 107(5), e4. https://doi.org/10.2105/AJPH.2017.303717
[12] National Academies of Sciences, Engineering, and Medicine; Health and Medicine Division; Board on Health Sciences Policy; Committee on Pain Management and Regulatory Strategies to Address Prescription Opioid Abuse; Phillips JK, Ford MA, Bonnie RJ, editors. Pain Management and the Opioid Epidemic: Balancing Societal and Individual Benefits and Risks of Prescription Opioid Use. Washington (DC): National Academies Press (US); 2017 Jul 13. 5, Evidence on Strategies for Addressing the Opioid Epidemic. Available from: https://www.ncbi.nlm.nih.gov/books/NBK458653/

[13] Howard G. Birnbaum, PhD, Alan G. White, PhD, Matt Schiller, BA, Tracy Waldman, BA, Jody M. Cleveland, MS, Carl L. Roland, PharmD, Societal Costs of Prescription Opioid Abuse, Dependence, and Misuse in the United States, Pain Medicine, Volume 12, Issue 4, April 2011, Pages 657-667, https://doi.org/10.1111/j. 1526-4637.2011.01075.x

[14] Center for Substance Abuse Treatment. Managing Chronic Pain in Adults With or in Recovery From Substance Use Disorders. Rockville (MD): Substance Abuse and Mental Health Services Administration (US); 2012. (Treatment Improvement Protocol (TIP) Series, No. 54.) 3, Chronic Pain Management. Available from: https://www.ncbi.nlm.nih.gov/books/NBK92054/

[15] Hendrée E. Jones et al. Naltrexone in the treatment of opioid-dependent pregnant women: the case for a considered and measured approach to research. https://doi.org/10.1111/j.1360-0443.2012.03811.x

[16] Lamont, P., \& Hunt, S. C. (2006). A twist on torsade: a prolonged QT interval on methadone. Journal of general internal medicine, 21(11), C9-C12. https://doi.org/10.1111/j.1525-1497.2006.00588.x

[17] Martin, J. A., Campbell, A., Killip, T., Kotz, M., Krantz, M. J., Kreek, M. J., McCarroll, B. A., Mehta, D., Payte, J. T., Stimmel, B., Taylor, T., Haigney, M. C., Wilford, B. B., \& Substance Abuse and Mental Health Services Administration (2011). QT interval screening in methadone maintenance treatment: report of a SAMHSA expert panel. Journal of addictive diseases, 30(4), 283306. https://doi.org/10.1080/10550887.2011.610710

[18] Alinejad, S., Kazemi, T., Zamani, N., Hoffman, R. S., \& Mehrpour, O. (2015). A systematic review of the cardiotoxicity of methadone. EXCLI journal, 14, 577600. https://doi.org/10.17179/excli2015-553

[19] Roger Chou et al. Methadone Safety: A Clinical Practice Guideline from the American Pain Society and College on Problems of Drug Dependence, in Collaboration With the Heart Rhythm Society. DOI:https://doi.org/10.1016/j.jpain.2014.01.494

[20] Alinejad, S., Kazemi, T., Zamani, N., Hoffman, R. S., \& Mehrpour, O. (2015). A systematic review of the cardiotoxicity of methadone. EXCLI journal, 14, 577 600. https://doi.org/10.17179/excli2015-553

[21] Tanaka, K., Kersten, J. R., \& Riess, M. L. (2014). Opioid-induced cardioprotection. Current pharmaceutical design, 20(36), 5696-5705. https://doi.org/10.2174/1381612820666140204120311

[22] Fryer, R. M., Wang, Y., Hsu, A. K., \& Gross, G. J. (2001). Essential activation of PKC-delta in opioidinitiated cardioprotection. American journal of physiology. Heart and circulatory physiology, 280(3), 
H1346-H1353.

https://doi.org/10.1152/ajpheart.2001.280.3.H1346

[23] Matthias Karck et al. Myocardial protection by ischemic preconditioning and $\delta$-opioid receptor activation in the isolated working rat heart. CARDIOPULMONARY SUPPORT AND PHYSIOLOGY (CSP). DOI:https://doi.org/10.1067/mtc.2001.116950

[24] Melo, Z., Ishida, C., Goldaraz, M. P., Rojo, R., \& Echavarria, R. (2018). Novel Roles of Non-Coding RNAs in Opioid Signaling and Cardioprotection. Noncoding RNA, 4(3), 22. https://doi.org/10.3390/ncrna4030022

[25] Wang, Q., Sun, Y., Li, J., Xing, W., Zhang, S., Gu, X., Feng, N., Zhao, L., Fan, R., Wang, Y., Yin, W., \& Pei, J. (2014). Quaternary ammonium salt of U50488H, a new $\kappa$-opioid receptor agonist, protects rat heart against ischemia/reperfusion injury. European journal of pharmacology, $\quad 737, \quad 177-184$. https://doi.org/10.1016/j.ejphar.2014.05.006

[26] Alinejad, S., Kazemi, T., Zamani, N., Hoffman, R. S., \& Mehrpour, O. (2015). A systematic review of the cardiotoxicity of methadone. EXCLI journal, 14, 577600. https://doi.org/10.17179/excli2015-553

[27] Gross, E. R., Hsu, A. K., \& Gross, G. J. (2009). Acute methadone treatment reduces myocardial infarct size via the delta-opioid receptor in rats during reperfusion. Anesthesia and analgesia, 109(5), 1395-1402. https://doi.org/10.1213/ANE.0b013e3181b92201

[28] Michael Marmor et al. Coronary artery disease and opioid use. The American Journal of Cardiology https://doi.org/10.1016/j.amjcard.2004.01.072

[29] Lemesle, F., Lemesle, F., Nicola, W., \& Pierre JonvilleBéra, A. (2010). First case of stress cardiomyopathy as a result of methadone withdrawal secondary to drug-drug interaction. The American journal of emergency medicine, 28(3), https://doi.org/10.1016/j.ajem.2009.07. 007

[30] Saiful, F. B., Lafferty, J., Jun, C. H., Teli, S., Duvvuri, S., Khattri, S., \& Bhat, T. (2011). Takotsubo cardiomyopathy due to iatrogenic methadone withdrawal. Reviews in cardiovascular medicine, 12(3), 164-167. 